\title{
Subtractive Cell-SELEX Selection of DNA Aptamers Binding Specifically and Selectively to Hepatocellular Carcinoma Cells with High Metastatic Potential
}

\author{
Hao Chen, ${ }^{1}$ Chun-Hui Yuan, ${ }^{2}$ Yi-Fei Yang, ${ }^{2}$ Chang-Qing Yin, ${ }^{1}$ Qing Guan, ${ }^{2}$ \\ Fu-Bing Wang, ${ }^{1}$ and Jian-Cheng $\mathrm{Tu}^{1}$ \\ ${ }^{1}$ Department of Laboratory Medicine and Center for Gene Diagnosis, Zhongnan Hospital of Wuhan University, \\ 169 Donghu Road, Wuchang District, Wuhan 430071, China \\ ${ }^{2}$ Department of Immunology, School of Basic Medical Sciences, Wuhan University, 185 Donghu Road, Wuchang District, \\ Wuhan 430071, China
}

Correspondence should be addressed to Fu-Bing Wang; wfb20042002@sina.com and Jian-Cheng Tu; jian_1999@yahoo.com

Received 17 November 2015; Revised 25 February 2016; Accepted 7 March 2016

Academic Editor: Melchiorre Cervello

Copyright (C) 2016 Hao Chen et al. This is an open access article distributed under the Creative Commons Attribution License, which permits unrestricted use, distribution, and reproduction in any medium, provided the original work is properly cited.

\begin{abstract}
Relapse and metastasis are two key risk factors of hepatocellular carcinoma (HCC) prognosis; thus, it is emergent to develop an early and accurate detection method for prognostic evaluation of HCC after surgery. In this study, we sought to acquire oligonucleotide DNA aptamers that specifically bind to HCC cells with high metastatic potential. Two HCC cell lines derived from the same genetic background but with different metastatic potential were employed: MHCC97L (low metastatic properties) as subtractive targets and HCCLM9 (high metastatic properties) as screening targets. To mimic a fluid combining environment, initial DNA aptamers library was firstly labelled with magnetic nanoparticles using biotin-streptavidin system and then applied for aptamers selection. Through 10-round selection with subtractive Cell-SELEX, six aptamers, LY-1, LY-13, LY-46, LY-32, LY-27/45, and LY-7/43, display high affinity to HCCLM9 cells and do not bind to MHCC97L cells, as well as other tumor cell lines, including breast cancer, lung cancer, colon adenocarcinoma, gastric cancer, and cervical cancer, suggesting high specificity for HCCLM9 cells. Thus, the aptamers generated here will provide solid basis for identifying new diagnostic targets to detect HCC metastasis and also may provide valuable clues for developing new targeted therapeutics.
\end{abstract}

\section{Introduction}

Hepatocellular carcinoma (HCC) is the second most common cause of cancer-related death worldwide, estimated to be responsible for nearly 746,000 deaths in 2012 (9.1\% of the total), and is a formidable public health challenge of China where $50 \%$ of the estimated 782,000 new cancer cases worldwide occurred $[1,2]$. In recent decades, great advancements have been achieved in the development of therapeutics for HCC; besides hepatic resection as a mainstay of HCC treatment, local ablative therapies have greatly improved patient survival when HCC is diagnosed at early stages and, of them, radiofrequency ablation (RFA) is considered the reference standard [3-5]. However, according to the data presented by WHO in 2012 (http://globocan.iarc.fr/Default.aspx), the prognosis for hepatocellular carcinoma is still very poor (overall ratio of mortality to incidence is 0.95$)[2,6,7]$.

As the two pivotal prognostic factors of HCC, postoperatively relapse and metastasis significantly shorted the survival time of surgically treated patients [8-10]. Currently, regular reexamination of serum alpha fetoprotein (AFP) level or contrast enhanced ultrasound (CEUS) still represents the two preferred diagnostic strategies in clinical examination to detect postoperatively relapse and metastasis [11]. However, with regard to early diagnosis of HCC, the positive rate of AFP is only $60-80 \%$ and often resulted in a false-positive result during pregnancy, as well as for active liver disease, embryonic tumor, and certain gastrointestinal tumors [12]. CEUS has been applied for more than ten years and has proved to be of great value in the management of HCC [13]. 
In most of the cases, HCC always shows earlier enhancement than the surrounding liver tissue; the detection rate in lesions larger than $2.1 \mathrm{~cm}$ is up to $92 \%-100 \%[14,15]$. However, when lesions are less than $1.0 \mathrm{~cm}$, the detection rate is lower than $67 \%$, and, apparently, CEUS has a relatively low ability to determine the smaller lesions of HCC in an early stage [16]. Thus, the identification of new tumor biomarkers involved in metastasis and recurrence is urgent in surveillance for HCC.

Since potential biomarkers can encompass various types of molecules ranging from glycolipids to proteins, thus, the strategy of Systematic Evolution of Ligands through Exponential Enrichment (SELEX) is ideally suited for the creation of biomarker, as aptamers generated by SELEX are capable of selective binding to any class of molecules [17]. Aptamers are synthetic, single-stranded oligonucleotides DNA or RNA that could fold into unique structures, including hairpin, fake festival, convex ring, and G-tetramer, to bind specifically to their target molecules [18]. Compared with antibodies, they possess several key advantages: smaller molecular weight (the average molecular weight of a DNA aptamer is about $25 \mathrm{kDa}$ ); without immunogenicity, greater specificity and affinity; and being easier to be economically produced and modified with multiple chemical molecules [18, 19]. Thus, aptamers have been widely used in cell imaging [20], clinical diagnosis, and targeted therapeutics [21-23].

Cell-SELEX derives from traditional SELEX process and uses whole living cells as target [24]. With the help of this technology, aptamers can be obtained even without prior knowledge of potential target molecules of cancer cells [25]. More importantly, Cell-SELEX-based selection of aptamers against cancer cells has been reported in different cancers, including leukemia, lung cancer, colon cancer, glioma, and ovarian cancer, as well as in HCC [25-28]. However, no information was given on the ability of aptamer to differentiate tumor cells with metastatic potential in HCC. In the present study, two HCC cell lines derived from the same genetic background but with different metastatic potential were employed: MHCC97L (low metastatic properties) as counterparts and HCCLM9 (high metastatic properties) as screening targets. Initial DNA aptamers library was labelled with magnetic nanoparticles and then applied for aptamers selection in a fluid compartment. Six aptamers selected by the Cell-SELEX display high affinity to HCCLM9 cells and do not bind to MHCC97L cells and other tumor cell lines, suggesting specificity for HCCLM9 cells. Thus, the aptamers generated here will provide solid basis for identifying new diagnostic targets to detect HCC metastasis.

\section{Materials and Methods}

2.1. Cell Lines and Reagents. MHCC97L cell and HCCLM9 cell were obtained from research center of Zhongnan Hospital, Wuhan University, as we previously described [29] and cultured in RPMI1640 (Gibco) containing 10\% FBS (Gibco) and 100 units/mL penicillin-streptomycin (Beyotime, Shanghai, China). Other cell lines were maintained at our laboratory. Salmon sperm DNA, yeast tRNA, and BSA were purchased from Roche (F. Hoffmann-La Roche Ltd., USA). Streptavidin-coated magnetic nanoparticles M-280
(Dynabeads) were used for modifying biotin-labelled singlestranded DNA (ssDNA) aptamers pool.

2.2. Random DNA Library and Primers. An initial ssDNA aptamer library (5' -ATCCAGAGTGACGCAGCA-N40-TGGACACGGT GGCTTAGT-3') consisting of 40 -base randomized sequences was synthesized (Invitrogen, Shanghai, China), where $\mathrm{N}$ represents a randomized nucleotide of either A, G, C, or T. Forward primer 1 ( $5^{\prime}$-ATCCAGAGTGACGCAGCA-3') and biotin-labelled reverse primer 2 ( $5^{\prime}$-ACTAA GCCACCGTGTCCA- $\left.3^{\prime}\right)$ were used for PCR amplification of the DNA library or to separate the singlestranded DNA by streptavidin-coated magnetic particles. The FAM-labelled forward primer 1 (SBS Genetech Co., Ltd.) was used to monitor progress of selection by flow cytometry (Beckman Coulter, USA).

2.3. Subtractive Cell-SELEX Procedure. Subtractive CellSELEX procedure was performed as we described previously [29]. Briefly, initial ssDNA aptamers library (8 nmol) was first dissolved in $1 \mathrm{~mL}$ precooled binding buffer (PBS $1 \mathrm{M}$, $\mathrm{MgCl}_{2} 0.1 \mathrm{mg} / \mathrm{mL}$, yeast tRNA $1 \mathrm{mg} / \mathrm{mL}$, BSA $0.1 \mathrm{mg} / \mathrm{mL}$, and Salmon sperm DNA $100 \mu \mathrm{g} / \mathrm{mL}$ ) and then incubated with adherent HCCLM9 cells for $1 \mathrm{~h}$ in an orbital shaker. After incubation, the cells were washed 3 times with binding buffer to remove unbound ssDNA aptamers. Adherent HCCLM9 cells were scraped off and resuspended in $500 \mu \mathrm{L}$ DNasefree deionized water. Cell suspension was then heated at $100^{\circ} \mathrm{C}$ for $5 \mathrm{~min}$ and centrifuged at $12000 \mathrm{~g}$ for $5 \mathrm{~min}$. Supernatant containing eluted ssDNA aptamers was collected and then amplified by PCR using biotin-labelled primer. The biotin-labelled ssDNA aptamers were then incubated with streptavidin-coated magnetic nanoparticles (Dynabeads, M280 Streptavidin, Invitrogen) and used for the next round of selection.

From the fourth round of selection, the selected ssDNA aptamers pool was firstly incubated with subtractive MHCC97L cells to perform subtractive selection and thus filtered out ssDNA aptamers that may bind to subtractive MHCC97L cells. The unbounded aptamers pool was specifically targeted to HCCLM9 cells and then incubated with HCCLM9 cells for positive selection. Furthermore, the target cell number and the concentration of ssDNA aptamers pool were gradually reduced with the selective round proceeding to 10 cycles.

2.4. The Binding Ability of Each Round of Enriched ssDNA Aptamers Pool. FAM-labelled ssDNA aptamers pools of 3, 5, 7 , and 9 rounds were incubated with target cells HCCLM9 or subtractive cells MHCC97L $\left(1 \times 10^{6}\right.$ for each $)$ in $500 \mu \mathrm{L}$ binding buffer on ice for $30 \mathrm{~min}$. Cells were washed twice after incubation and the fluorescence intensity was determined by flow cytometry. The FAM-labelled control aptamer NK8 (bound to Mycobacterium tuberculosis) was used as a negative control $[29,30]$.

2.5. Clones Selection and Sequencing. The ssDNA aptamers pool of 9 rounds was amplified by PCR to obtain 
double-stranded DNAs (dsDNAs) using unmodified primers and then cloned into a $\mathrm{T}$ vector (Invitrogen). The recombinant plasmid was then transformed into $E$. coli $\mathrm{DH} 5 \alpha$ and randomly selected 50 clones using blue-white selection. The selected 50 clones were sequenced by Invitrogen Co., Ltd. (Shanghai, China) and designated as LY1 to LY50.

2.6. Sequence Alignments and Secondary Structures Analysis of Aptamers. DNAMAN software version 6.0 (Lynnon Biosoft, CA, USA) was used for sequence analysis and alignments. RNA structure (version 4.5, University of Rochester Medical Center) and MEME online analysis software version 4.10.2 (http://meme-suite.org/tools/meme) were used to estimate the secondary structures of sequenced aptamers.

2.7. Fluorescence Imaging of the Selected Aptamers Bound to HCCLM9 Cells. The FAM-labelled aptamers were synthesized by SBS Genetech Co., Ltd. (Shanghai, China). HCCLM9 cells were cultured in chamber slides overnight. The FAMlabelled individual aptamer was incubated with cell monolayer in chamber slides in binding buffer on $4^{\circ} \mathrm{C}$ for $30 \mathrm{~min}$. After washing 3 times with PBS, the cells were imaged with Olympus BX51 fluorescence microscope (Olympus, Tokyo, Japan).

2.8. Determination of the Dissociation Constants $\left(K_{d}\right)$ of Individual ssDNA Aptamers. To determine the $K_{d}$ value of the six selected aptamers, HCCLM9 cells $\left(1 \times 10^{6}\right)$ were incubated with various concentrations of FAM-labelled aptamers in binding buffer at $4^{\circ} \mathrm{C}$ for $30 \mathrm{~min}$ and the fluorescence intensity was determined by flow cytometry. The mean fluorescence intensity (MFI) of negative control aptamer NK8 was subtracted from that of each aptamer with the target cells to determine the specific binding of each aptamer. Then, the equilibrium dissociation constants $\left(K_{d}\right)$ of each aptamer were determined by nonlinear regression for one-site binding according to the equation: $Y=B_{\max } \times X /\left(K_{d}+X\right)$ using GraphPad Prism version 5.0 (GraphPad Software, Inc.).

2.9. Specificity Analysis of Selected Aptamers. To determine the cell specificity of the selected aptamers, human cancer cell lines including HCC cell lines MHCC97L, HepG2, and Huh-7, breast cancer cell line MDA-MB-231, lung cancer cell line H1299, colon adenocarcinoma cell line SW48, gastric cancer cell line MGC803, and cervical cancer cell line HeLa were used to test the specific binding affinity with fluorescent microcopy assay.

\section{Results}

3.1. Aptamers Selection and Binding Affinity Analysis. Subtractive Cell-SELEX was performed using HCC cell line HCCLM9 cells (with high metastatic properties) as the target and MHCC97L cells (low metastatic properties) as subtractive target, respectively, for the selection of metastatic-specific aptamers. HCCLM9 and MHCC97L were differentiated from MHCC97 cells as shown in our previous studies $[29,31]$. In order to get aptamers with high specificity, 10 rounds of selection were performed. And with increasing rounds of enrichment, the number of target cells, ssDNA concentration, and the incubation time were gradually decreased, while the number of washing times was increased to reinforce the selective pressure. In the selection procedure, we amplified the aptamer pools of 2, 4, 6, 8, 9, and 10 cycles and analyzed them with agarose gel electrophoresis. With the selection cycles increased, the gray value of corresponding pool was gradually increased in the same PCR cycle and was the highest in 9 cycles. In 10 cycles of selection, the corresponding gray value was decreased compared with 9 cycles; thus, we speculated that aptamers of 9 cycles were optimal and included ssDNA aptamers that specifically bind to HCCLM9 cells. Simultaneously, we compared minimum PCR amplification cycles of each round pool; the minimum PCR amplification cycles in 9 rounds were 8 , significantly lower than other rounds; these results further confirmed that 9 rounds of enrichment were optimal.

Next, we labelled these selected rounds of aptamers with FAM and then incubated them with HCCLM9 cells and MHCC97L cells to examine the specific binding ability using flow cytometry. Relative fluorescence intensity was calculated as $\left(F_{\text {aptamer }}-F_{\text {cell }}\right) /\left(F_{\text {control }}-F_{\text {cell }}\right)$. With increasing selective rounds, the relative fluorescence intensity of aptamer pool bound on HCCLM9 cells gradually increased and reached the highest value in 9 rounds, while the relative fluorescence intensity of MHCC97L cells showed no discernible change (Figure 1). Therefore, it was evident that the pool of ssDNA aptamers in 9 cycles has preferential and specific binding to HCCLM9 cells.

3.2. Selection, Sequencing, and Structure Prediction of Aptamers. Then, the ssDNA aptamers pool in 9 cycles was amplified into double strand and cloned into $T$ vector and transformed into E. coli $5 \alpha$. Next, fifty clones were randomly selected using blue-white selection and subjected to sequencing analysis. Among these 50 clones, 10 clones were discarded with no or multiple sequences detected. 23 clones yielding the same sequence are marked as LY-1, 9 clones yielding the same sequence are marked as LY-13, 2 clones yielding the same sequence are marked as LY-7/43, another 2 clones yielding the same sequence are marked as LY-27/45, and the rest 2 clones each yielding one sequence are marked as LY-32 and LY-46 (Figure 2(a)).

According to the sequencing result, we further analyzed the sequence homology and structure prediction of these six selected aptamers. The greatest percentage of homology existed between LY-32 and LY-46; the second was LY-1 and LY-13 (Figure 2(b)). Aptamers that are contained within homologous groups are likely to have been strongly preferentially enriched during the selection process. All of the six aptamers showed a tendency towards G-richness, T-richness, or both (Figure 2(a)). The secondary-structural analysis of the six chosen aptamers was shown in Figure 2(c).

3.3. Specific Binding Affinity of the Six Selected Aptamers. To further confirm the specific binding affinity of these six selected aptamers to HCCLM9 cells, we firstly labelled 

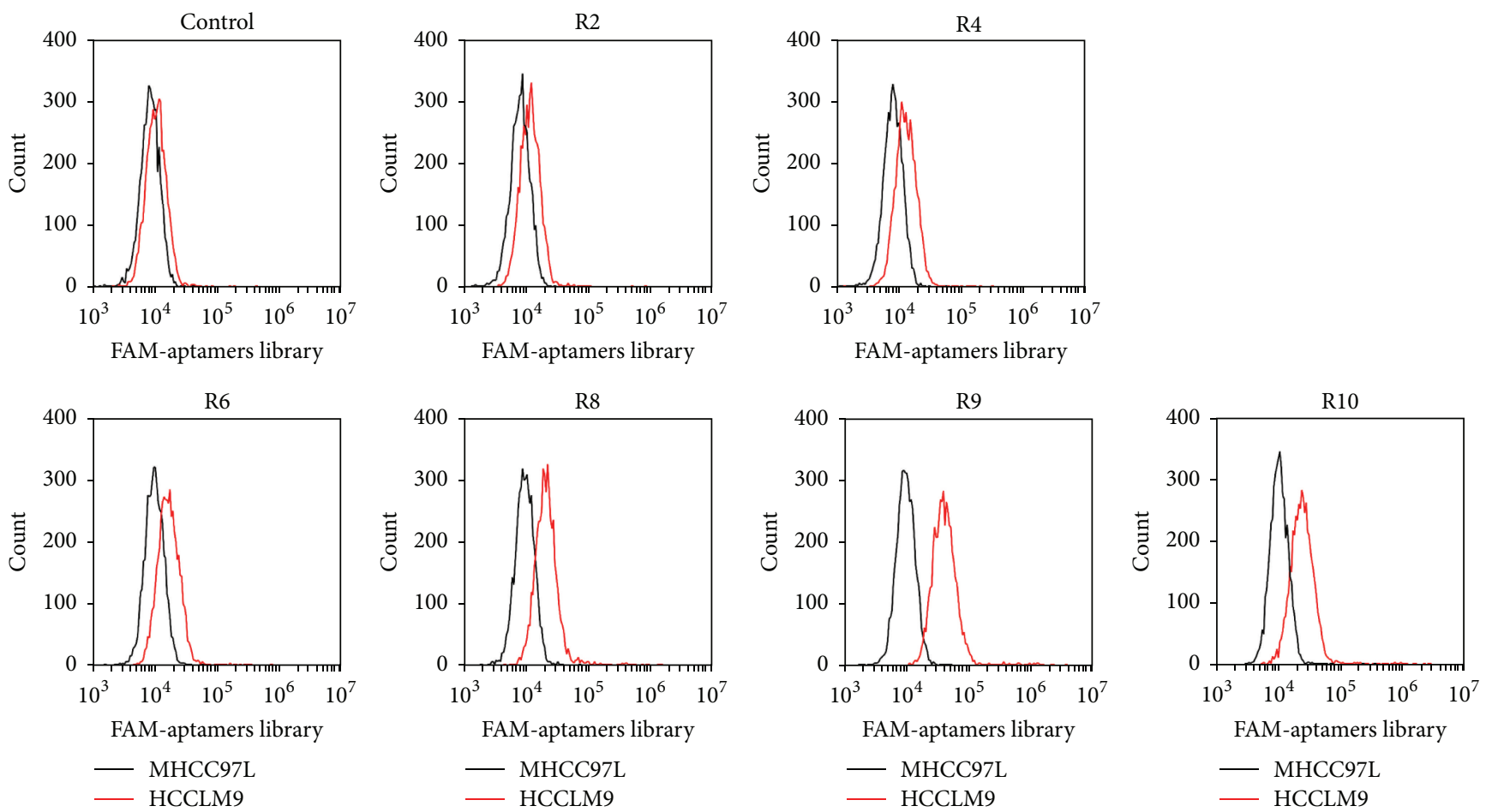

(a)

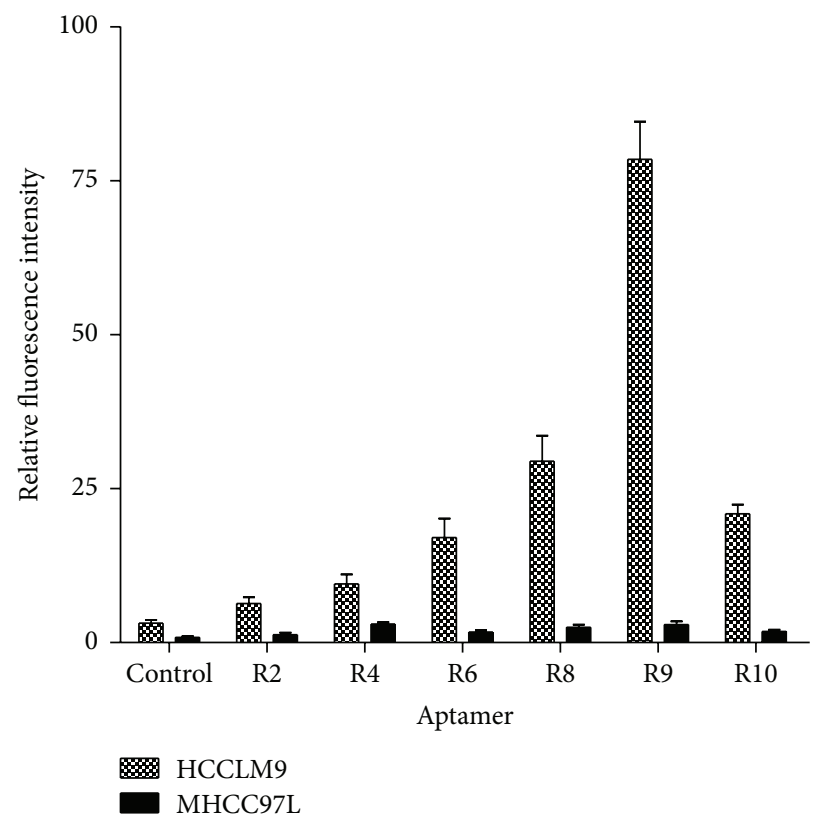

(b)

FIGURE 1: (a) The specific binding ability of aptamers library in selected rounds with HCCLM9 (target cells) and MHCC97L (subtractive cells) was analyzed by flow cytometry. With increasing rounds of enrichment, significant increases in fluorescence intensity were detected on HCCLM9 cells but not on MHCC97L and reached the peak at the ninth round. (b) Fluorescence shift was calculated using the equation $\left(F_{\text {aptamer }}-F_{\text {cell }}\right) /\left(F_{\text {library }}-F_{\text {cell }}\right)$, where $F_{\text {aptamer }}, F_{\text {library }}$, and $F_{\text {cell }}$ refer to the fluorescence of the selected aptamers pool, initial library, and the cell background, respectively.

aptamers with FAM at the $5^{\prime}$ end. And then their binding affinity to HCCLM9 cells was evaluated with fluorescent microcopy assay. The percentage of the cells with fluorescence above the set threshold was used to evaluate the binding capacity of the aptamer to the cells [32]. All six aptamers were significantly bound to the target HCCLM9 cells compared to the ssDNA library; particularly, LY-1 and LY-13 had the highest binding capacity (Figure 3(a)). Moreover, all six 


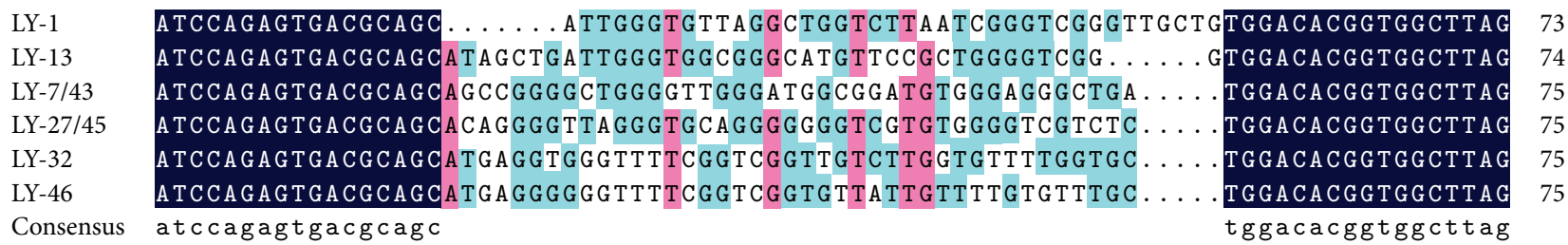

(a)

\begin{tabular}{lcccccc}
\multicolumn{7}{l}{ Homology matrix of 6 sequences } \\
& LY-1 & LY-13 & LY-7/43 & LY-27/45 & LY-32 & LY-46 \\
LY-1 & $100 \%$ & & & & & \\
LY-13 & $83.60 \%$ & $100 \%$ & & & & \\
LY-7/43 & $67.20 \%$ & $67.20 \%$ & $100 \%$ & & & \\
LY-27/45 & $74.60 \%$ & $74.60 \%$ & $73.10 \%$ & $100 \%$ & & \\
LY-32 & $68.70 \%$ & $68.70 \%$ & $62.70 \%$ & $68.70 \%$ & $100 \%$ & \\
LY-46 & $65.70 \%$ & $62.70 \%$ & $64.20 \%$ & $68.70 \%$ & $86.60 \%$ & $100 \%$
\end{tabular}

(b)
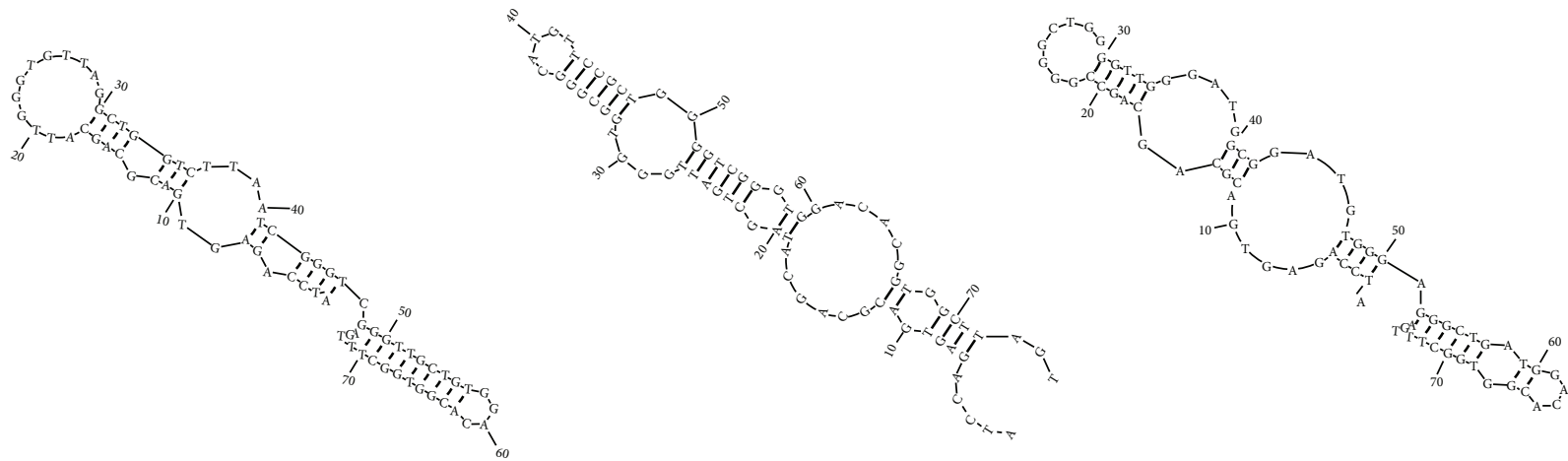

LY-1

LY-13

LY-7/43
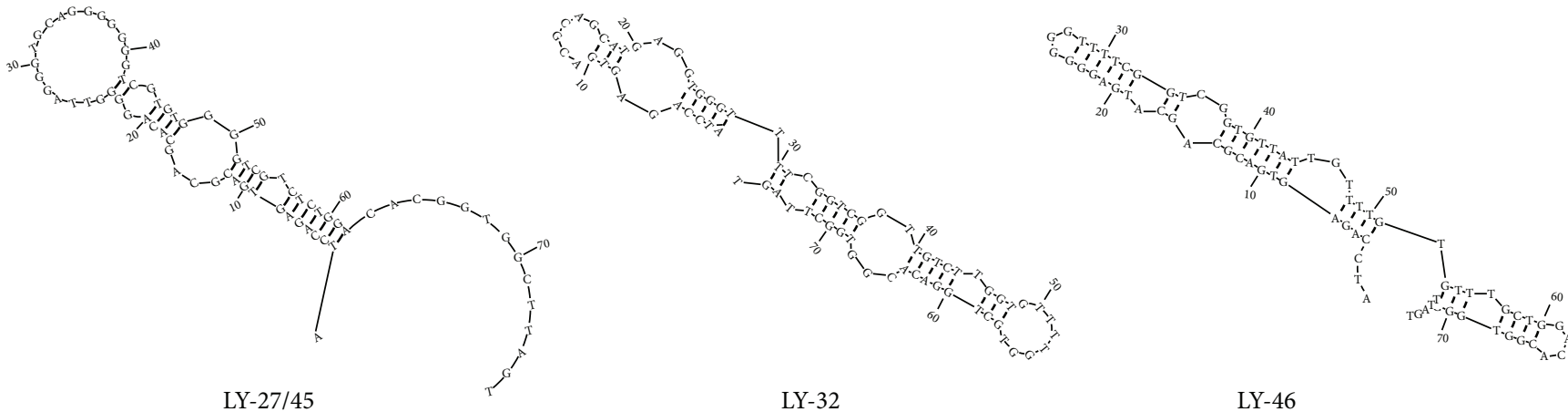

LY-32

LY-46

(c)

FIgURE 2: The homology analysis and secondary structure prediction of six aptamers. (a) Sequence alignment of six aptamers; all of the six aptamers showed a tendency towards G-richness, T-richness, or both. (b) Homology analysis of six aptamers; the greatest percentage of homology existed between LY-32 and LY-46; the second was LY-1 and LY-13. (c) Secondary structure prediction of six aptamers by RNA structure software.

aptamers displayed high binding affinity to HCCLM9 cells with $K_{d}$ values in the tiny range from 167.3 to $369.7 \mathrm{nM}$ (Figure 3(b), Table 1), and LY-1 exhibited the highest binding affinity with a $K_{d}$ value of $167.3 \pm 30.2 \mathrm{nM}$.

Next, to test the binding specificity of these six aptamers, different HCC cells (MHCC97L, Huh-7, and HepG2), human lung cancer cell line H1299, human colon adenocarcinoma cell line SW48, human gastric cancer cell line MGC803, human cervical cancer cell line HeLa, human breast cancer cell line MDA-MB-231, and human peripheral WBC were used. As concluded in Table 2, all six aptamers exhibited specific binding affinity to HCCLM9 cells to different extent. LY1 and LY-13 also recognized other HCC cell lines, including MHCC97L, Huh-7, and HepG2 cells, while the rest aptamers only recognized one kind of the other HCC cell lines, beside HCCLM9 cells, and control aptamer NK8 showed no 


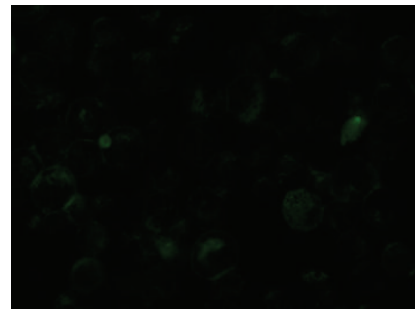

ssDNA library

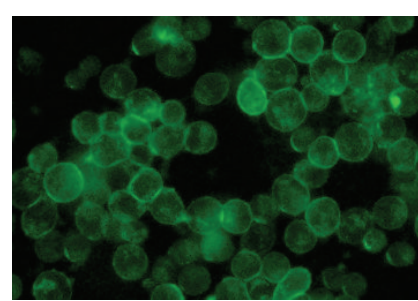

LY-1

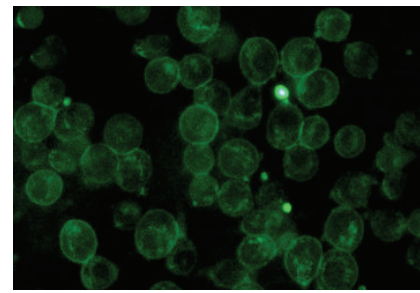

LY-32

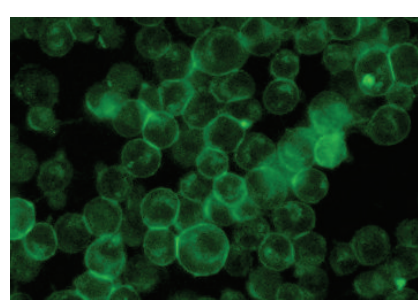

LY-13

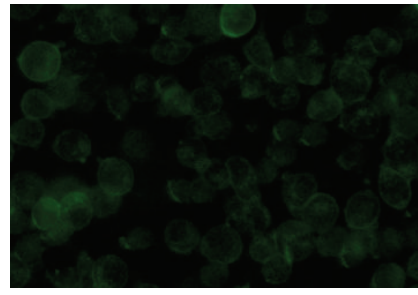

LY-7/43

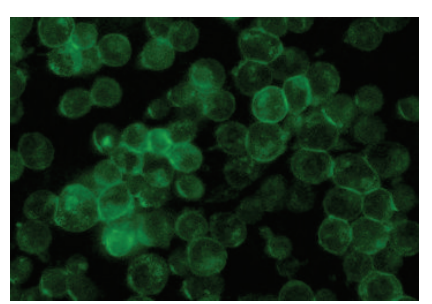

LY-27/45

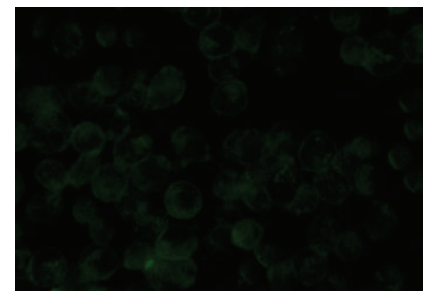

LY-46

(a)
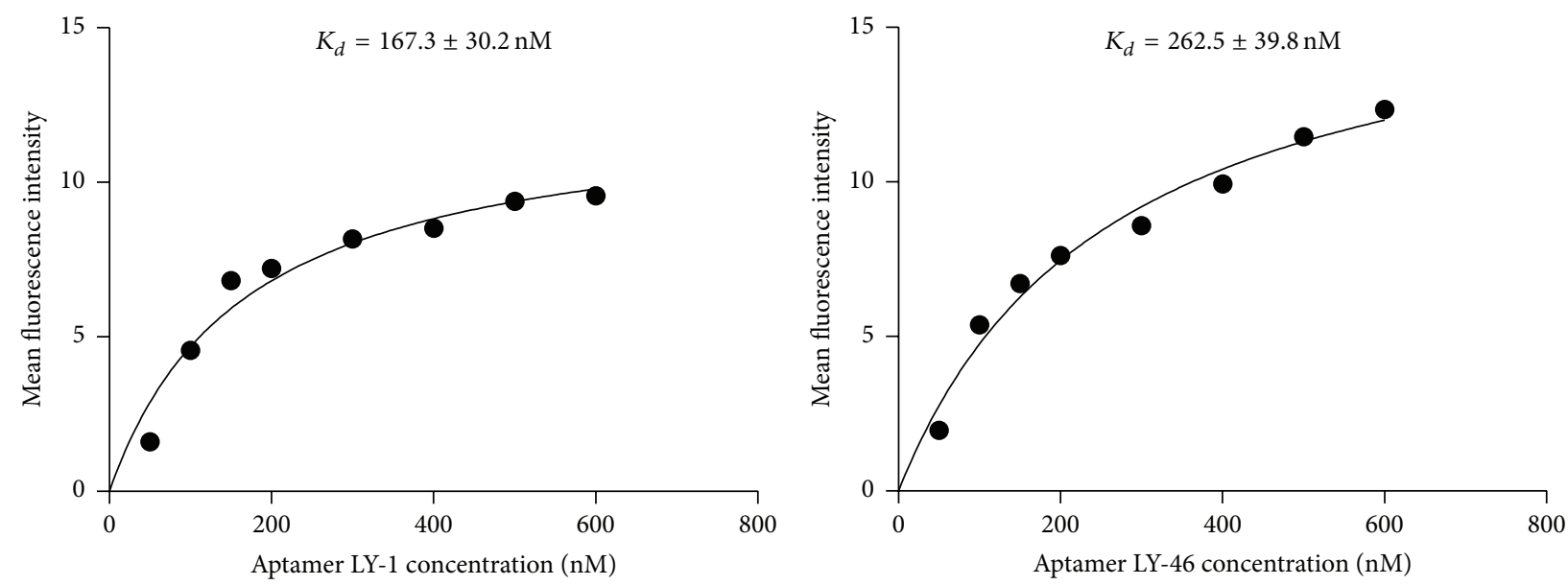

(b)

FiguRE 3: The specific binding affinity of these six selected aptamers to HCCLM9 cells. (a) The fluorescence imaging of six selected aptamers and initial ssDNA library control bound to HCCLM9 cells. Compared with the control group, all six selected aptamers were obviously bound to the membrane of target HCCLM9 cells; particularly, LY-1 and LY-13 had the highest binding capacity. (b) Binding curve of aptamers LY-1 and LY-46 with target HCCLM9 cells. Cells were incubated with increasing concentrations of FAM-labelled aptamers and the fluorescence intensity (MFI) was detected by flow cytometry. After subtracting the MFI of negative aptamer control (NK8), the MFI of FAM-aptamer bound target cells was used to calculate the $K_{d}$ value of each aptamer.

detectable binding affinity to any kinds of cells. However, these six aptamers exhibited no detectable recognition of any other tumor cell lines and normal white blood cells (WBC), revealing that the six aptamers specifically targeted metastatic HCC cells with high affinity, especially LY-1 and LY-13.

\section{Discussion}

When developing new molecular probes for cancers, CellSELEX has advantages over traditional protein-SELEX, as it could be fulfilled just depending on expressive difference of cell surface molecules between target cells and control cells, even without the need to figure out the detailed molecular characteristics of target cells [32]. More importantly, surface molecules of target cells can maintain native folding and
TABLE 1: $K_{d}$ s of six selected aptamers of the HCCLM9 cells.

\begin{tabular}{lc}
\hline Aptamer & $K_{d}$ value $(\mathrm{nM})$ \\
\hline LY-1 & $167.3 \pm 30.2$ \\
LY-13 & $185.6 \pm 28.3$ \\
LY-27/45 & $303.6 \pm 34.5$ \\
LY-32 & $245.7 \pm 44.4$ \\
LY-7/43 & $369.7 \pm 46.3$ \\
LY-46 & $262.5 \pm 39.8$ \\
\hline
\end{tabular}

glycosylation states and an improved specificity can further be achieved by performing an additional subtractive step [25]. The target molecules of aptamers selected by subtractive CellSELEX may be the different compositions between tumor 
TABLE 2: The binding specificity of six aptamers to different cell lines.

\begin{tabular}{|c|c|c|c|c|c|c|c|}
\hline \multirow{2}{*}{ Cell lines } & \multicolumn{7}{|c|}{ Aptamers } \\
\hline & LY-1 & LY-13 & LY-27/45 & LY-32 & LY-7/43 & LY-46 & Control \\
\hline HCCLM9 & ++++ & ++++ & ++ & ++ & + & + & - \\
\hline MHCC97L & + & + & - & + & - & - & - \\
\hline Huh7 & + & + & - & - & + & - & - \\
\hline HepG2 & + & + & + & - & - & + & - \\
\hline H1299 & - & - & - & - & - & - & - \\
\hline SW480 & - & - & - & - & - & - & - \\
\hline MGC803 & - & - & - & - & - & - & - \\
\hline $\mathrm{HeLa}$ & - & - & - & - & - & - & - \\
\hline MDA-MB-231 & - & - & - & - & - & - & - \\
\hline $\mathrm{WBC}$ & - & - & - & - & - & - & - \\
\hline
\end{tabular}

cells and normal counterparts $[33,34]$. Thus, studies focused on these different compositions may provide valuable clues for the development of new tumor-specific biomarkers or therapeutic targets.

In recent years, subtractive Cell-SELEX has been widely applied in identifying new tumor biomarker. In gastric cancer, Cao et al. [35] successfully selected ssDNA aptamer cy-apt 20 by using human gastric cancer AGS cells as target cells and normal gastric epithelium GES-1 cells as subtractive cells. In clinical reexamination, cy-apt 20 further showed great diagnostic and therapeutic potential of gastric cancer. Shangguan et al. [36] developed 6 Cell-SELEX-generated aptamers against neoplastic cells; among these aptamers, sgd5 recognized only its target Toledo cells, while sgc3, sgd2, sgd3, sgc4, and sgc8, selected from a T-cell acute leukemia (T-ALL) cell line CCRF-CEM cells, identified all of the cultured T-ALL cell lines. Furthermore, sgc8, sgc3, and sgd3 showed good selectivity toward T-ALL cells and neoplastic cells in patient samples, while almost not binding to normal hematopoietic cells or lymphoma and myeloma cells. Importantly, by using differential Cell-SELEX, Cerchia et al. developed five aptamers (GL36, GL35, GL44, GL43, and GL21) which specifically distinguished U87MG glioma cells from the less malignant cell line T98G and other nonrelated cancer cell types [28].

Metastasis is the major obstacle that resulted in the failure treatment and recurrence of HCC; thus, to identify new tumor biomarkers with great potential to reflect HCC-related metastasis has been a hot research point in recent years [37-39]. Gkretsi and Bogdanos [8] firstly demonstrated that migfilin promoted HCC metastasis in vitro and may serve as a potential therapeutic target. Aptamer is a new kind of biomarkers, which has been widely used in diagnosis of multiple cancers [40-42]. Kashefi-Kheyrabadi et al. [43] and Sun et al. $[44,45]$ coupled aptamer TLS1la with electrochemical biosensor which effectively differentiated HCC HepG2 cells from lung cancer or prostate cancer cells and provided a new target for early diagnosis. While most aptamers used HepG2 cells as selective target and normal liver cells as subtractive cells, thus, these aptamers could be only used for early diagnosis and showed no help in indicating metastasis of HCC [25]. Herein, we used high metastatic HCCLM9 cells as the target cells and the less metastatic MHCC97L cells as the negative cells to perform subtractive Cell-SELEX under strict selection conditions and thereby generated a panel of six aptamers that can specifically bind to HCC cells with high metastatic potential. It is worth noting that HCCLM9 cell line was derived from MHCC97L cells but with high metastatic potential, the different compositions between these two cell lines may only relate to metastasis [46, 47], and, thus, aptamers generated from these two cell lines may specifically recognize metastatic-related surface molecules expressed on HCC cells and could be potential biomarkers to predict metastasis of HCC.

In aptamers selection procedure, we randomly selected 50 clones of the nine rounds of ssDNA aptamers pool. Aptamers sequencing and alignments analysis revealed that five mutual motifs existed in these selected aptamers and continuous Grich motif abundantly existed in our six selected aptamers, which showed high binding affinity to HCCLM9 cells. This result suggested that aptamers in our initial ssDNA pool were specifically enriched by subtractive Cell-SELEX technique [48]. These six aptamers will further be subdivided into four families according to mutual motifs existing in secondary structure analysis, and continuous G-rich motif form stemloops in these aptamers. Next, we designated these aptamers as LY-1, LY-13, LY-46, LY-32, LY-27/45, and LY-7/43 and further labelled these six aptamers with FAM to examine the specific binding ability of individual aptamer to less metastatic HCC cells (MHCC97L, HepG2, and Huh-7) or different tumor cell lines, including breast cancer, lung cancer, colon adenocarcinoma, gastric cancer, and cervical cancer. Though the $K_{d}$ values of six selected aptamers may be higher than the aptamer reported by other researchers [32, 49], the six selected aptamers showed high specificity and sensitivity as potential molecular probes. And no matter whether aptamers are modified with fluorophore or magnetic nanoparticle, the specific binding ability of aptamers to high metastatic HCC cells was not changed; this may hint that these aptamers could be further conjugated with new types of luminescent or imaging materials and thus be developed into promising molecular probes for early prediction of HCC metastasis. 


\section{Competing Interests}

The authors declare that there are no competing interests regarding the publication of this paper.

\section{Authors' Contributions}

Hao Chen and Chun-Hui Yuan contributed equally to this work.

\section{Acknowledgments}

This work was supported by National Natural Science Foundation of China (no. 81371897) and research grants funded by Independent Research Fund Program of Wuhan University (no. 2042014kf0241). This work was also funded by "351 Talent Project (Luojia Young Scholars)” of Wuhan University and National Program on Key Basic Research Project (973 Program, no. 2012CB720600-05).

\section{References}

[1] J. Ferlay, I. Soerjomataram, R. Dikshit et al., "Cancer incidence and mortality worldwide: sources, methods and major patterns in GLOBOCAN 2012," International Journal of Cancer, vol. 136, no. 5, pp. E359-E386, 2015.

[2] M. J. Song and S. H. Bae, "Newer treatments for advanced hepatocellular carcinoma," Korean Journal of Internal Medicine, vol. 29, no. 2, pp. 149-155, 2014.

[3] A. Cucchetti, F. Piscaglia, M. Cescon, G. Ercolani, and A. D. Pinna, "Systematic review of surgical resection vs radiofrequency ablation for hepatocellular carcinoma," World Journal of Gastroenterology, vol. 19, no. 26, pp. 4106-4118, 2013.

[4] T. Kamiyama, K. Nakanishi, H. Yokoo et al., "Perioperative management of hepatic resection toward zero mortality and morbidity: analysis of 793 consecutive cases in a single institution," Journal of the American College of Surgeons, vol. 211, no. 4, pp. 443-449, 2010.

[5] A. Cucchetti, M. Zanello, M. Cescon et al., "Improved diagnostic imaging and interventional therapies prolong survival after resection for hepatocellular carcinoma in cirrhosis: the university of Bologna experience over 10 years," Annals of Surgical Oncology, vol. 18, no. 6, pp. 1630-1637, 2011.

[6] Y.-P. Wang and D.-X. Tang, "Expression of yes-associated protein in liver cancer and its correlation with clinicopathological features and prognosis of liver cancer patients," International Journal of Clinical and Experimental Medicine, vol. 8, no. 1, pp. 1080-1086, 2015.

[7] M. Lempinen, I. Lyytinen, A. Nordin et al., "Prognostic value of serum MMP-8, -9 and TIMP-1 in patients with hepatocellular carcinoma," Annals of Medicine, vol. 45, no. 7, pp. 482-487, 2013.

[8] V. Gkretsi and D. P. Bogdanos, "Experimental evidence of Migfilin as a new therapeutic target of hepatocellular carcinoma metastasis," Experimental Cell Research, vol. 334, no. 2, pp. 219227, 2015.

[9] L.-Y. Yang, W.-Q. Lu, G.-W. Huang, and W. Wang, "Correlation between CD105 expression and postoperative recurrence and metastasis of hepatocellular carcinoma," BMC Cancer, vol. 6, article 110, 2006.

[10] D.-P. Ou, L.-Y. Yang, G.-W. Huang, Y.-M. Tao, X. Ding, and Z.G. Chang, "Clinical analysis of the risk factors for recurrence of HCC and its relationship with HBV,' World Journal of Gastroenterology, vol. 11, no. 14, pp. 2061-2066, 2005.

[11] L. Wang, M. Yao, Z. Dong, Y. Zhang, and D. Yao, "Circulating specific biomarkers in diagnosis of hepatocellular carcinoma and its metastasis monitoring," Tumor Biology, vol. 35, no. 1, pp. 9-20, 2014.

[12] Y. J. Zhao, Q. Ju, and G. C. Li, “Tumor markers for hepatocellular carcinoma," Molecular and Clinical Oncology, vol. 1, no. 4, pp. 593-598, 2013.

[13] S. G. Zheng, H. X. Xu, and L. N. Liu, "Management of hepatocellular carcinoma: the role of contrast-enhanced ultrasound," World Journal of Radiology, vol. 6, no. 1, pp. 7-14, 2014.

[14] H.-X. Xu, X.-Y. Xie, M.-D. Lu et al., "Contrast-enhanced sonography in the diagnosis of small hepatocellular carcinoma $\leq 2 \mathrm{~cm}$," Journal of Clinical Ultrasound, vol. 36, no. 5, pp. 257266, 2008.

[15] L. Bolondi, S. Gaiani, N. Celli et al., "Characterization of small nodules in cirrhosis by assessment of vascularity: the problem of hypovascular hepatocellular carcinoma," Hepatology, vol. 42, no. 1, pp. 27-34, 2005

[16] M. H. Chen, Y. Dai, K. Yan et al., "The role of contrast-enhanced ultrasound on the diagnosis of small hepatocellular carcinoma $(</=3 \mathrm{~cm})$ in patients with cirrhosis," Hepatology Research, vol. 35, no. 4, pp. 281-288, 2006.

[17] J. A. Phillips, D. Lopez-Colon, Z. Zhu, Y. Xu, and W. Tan, "Applications of aptamers in cancer cell biology," Analytica Chimica Acta, vol. 621, no. 2, pp. 101-108, 2008.

[18] R. D. Walters, D. T. McSwiggen, J. A. Goodrich, and J. F. Kugel, "Selection and characterization of a DNA aptamer that can discriminate between cJun/cJun and cJun/cFos," PLoS ONE, vol. 9, no. 6, Article ID e101015, 2014.

[19] S. J. Lee, B. Adler, S. Ekström et al., "Aptamer/ISET-MS: a new affinity-based MALDI technique for improved detection of biomarkers," Analytical Chemistry, vol. 86, no. 15, pp. 76277634,2014

[20] M. Chen, S. Bi, X. Jia, and P. He, "Aptamer-conjugated biobar-code $\mathrm{Au}-\mathrm{Fe}_{3} \mathrm{O}_{4}$ nanoparticles as amplification station for electrochemiluminescence detection of tumor cells," Analytica Chimica Acta, vol. 837, pp. 44-51, 2014.

[21] Z. Han, X. Wang, C. Heng et al., "Synergistically enhanced photocatalytic and chemotherapeutic effects of aptamer-functionalized $\mathrm{ZnO}$ nanoparticles towards cancer cells," Physical Chemistry Chemical Physics, vol. 17, no. 33, pp. 21576-21582, 2015.

[22] M. Yang, G. Jiang, W. Li et al., "Developing aptamer probes for acute myelogenous leukemia detection and surface protein biomarker discovery," Journal of Hematology and Oncology, vol. 7, article 5, 2014.

[23] X.-L. Tang, Y.-X. Zhou, S.-M. Wu, Q. Pan, B. Xia, and X.-L. Zhang, "CFP10 and ESAT6 aptamers as effective Mycobacterial antigen diagnostic reagents," Journal of Infection, vol. 69, no. 6, pp. 569-580, 2014.

[24] K.-T. Guo, A. Paul, C. Schichor, G. Ziemer, and H. P. Wendel, "Cell-SELEX: novel perspectives of aptamer-based therapeutics," International Journal of Molecular Sciences, vol. 9, no. 4, pp. 668-678, 2008.

[25] K. Ninomiya, K. Kaneda, S. Kawashima, Y. Miyachi, C. Ogino, and N. Shimizu, "Cell-SELEX based selection and characterization of DNA aptamer recognizing human hepatocarcinoma," Bioorganic and Medicinal Chemistry Letters, vol. 23, no. 6, pp. 1797-1802, 2013. 
[26] D. Shangguan, Y. Li, Z. Tang et al., "Aptamers evolved from live cells as effective molecular probes for cancer study," Proceedings of the National Academy of Sciences of the United States of America, vol. 103, no. 32, pp. 11838-11843, 2006.

[27] H. W. Chen, C. D. Medley, K. Sefah et al., "Molecular recognition of small-cell lung cancer cells using aptamers," ChemMedChem, vol. 3, no. 6, pp. 991-1001, 2008.

[28] L. Cerchia, C. L. Esposito, A. H. Jacobs, B. Tavitian, and V. de Franciscis, "Differential SELEX in human glioma cell lines," PLoS ONE, vol. 4, no. 11, Article ID e7971, 2009.

[29] F.-B. Wang, Y. Rong, M. Fang et al., "Recognition and capture of metastatic hepatocellular carcinoma cells using aptamerconjugated quantum dots and magnetic particles," Biomaterials, vol. 34, no. 15, pp. 3816-3827, 2013.

[30] F. Chen, J. Zhou, F. Luo, A.-B. Mohammed, and X.-L. Zhang, "Aptamer from whole-bacterium SELEX as new therapeutic reagent against virulent Mycobacterium tuberculosis," Biochemical and Biophysical Research Communications, vol. 357, no. 3, pp. 743-748, 2007.

[31] Y. Li, Z. Y. Tang, S. L. Ye et al., "Establishment of a hepatocellular carcinoma cell line with unique metastatic characteristics through in vivo selection and screening for metastasis-related genes through cDNA microarray," Journal of Cancer Research and Clinical Oncology, vol. 129, no. 1, pp. 43-51, 2003.

[32] W.-M. Li, T. Bing, J.-Y. Wei, Z.-Z. Chen, D.-H. Shangguan, and J. Fang, "Cell-SELEX-based selection of aptamers that recognize distinct targets on metastatic colorectal cancer cells," Biomaterials, vol. 35, no. 25, pp. 6998-7007, 2014.

[33] L.-Y. Hung, C.-H. Wang, K.-F. Hsu, C.-Y. Chou, and G.-B. Lee, "An on-chip Cell-SELEX process for automatic selection of high-affinity aptamers specific to different histologically classified ovarian cancer cells," Lab on a Chip, vol. 14, no. 20, pp. 4017-4028, 2014.

[34] J. C. Graham and H. Zarbl, "Use of cell-SELEX to generate DNA aptamers as molecular probes of HPV-associated cervical cancer cells," PLoS ONE, vol. 7, no. 4, Article ID e36103, 2012.

[35] H.-Y. Cao, A.-H. Yuan, W. Chen, X.-S. Shi, and Y. Miao, "A DNA aptamer with high affinity and specificity for molecular recognition and targeting therapy of gastric cancer," BMC cancer, vol. 14, article 699, 2014.

[36] D. Shangguan, Z. C. Cao, Y. Li, and W. Tan, "Aptamers evolved from cultured cancer cells reveal molecular differences of cancer cells in patient samples," Clinical Chemistry, vol. 53, no. 6, pp. 1153-1155, 2007.

[37] S. K. Yoon, N. K. Lim, S.-A. Ha et al., "The human cervical cancer oncogene protein is a biomarker for human hepatocellular carcinoma," Cancer Research, vol. 64, no. 15, pp. 5434-5441, 2004.

[38] H.-W. L. Hann, J. Lee, A. Bussard et al., "Preneoplastic markers of hepatitis B virus-associated hepatocellular carcinoma," Cancer Research, vol. 64, no. 20, pp. 7329-7335, 2004.

[39] J. A. Marrero, P. R. Romano, O. Nikolaeva et al., "GP73, a resident Golgi glycoprotein, is a novel serum marker for hepatocellular carcinoma," Journal of Hepatology, vol. 43, no. 6, pp. 1007-1012, 2005.

[40] L. Meng, L. Yang, X. Zhao et al., "Targeted delivery of chemotherapy agents using a liver cancer-specific aptamer," PloS ONE, vol. 7, no. 4, Article ID e33434, 2012.

[41] X. Wu, Z. Zhao, H. Bai et al., "DNA aptamer selected against pancreatic ductal adenocarcinoma for in vivo imaging and clinical tissue recognition," Theranostics, vol. 5, no. 9, pp. 985994, 2015.
[42] H. Shi, W. Cui, X. He et al., "Whole Cell-SELEX aptamers for highly specific fluorescence molecular imaging of carcinomas in vivo," PLoS ONE, vol. 8, no. 8, Article ID e70476, 2013.

[43] L. Kashefi-Kheyrabadi, M. A. Mehrgardi, E. Wiechec, A. P. F. Turner, and A. Tiwari, "Ultrasensitive detection of human liver hepatocellular carcinoma cells using a label-free aptasensor," Analytical Chemistry, vol. 86, no. 10, pp. 4956-4960, 2014.

[44] D. Sun, J. Lu, Z. Chen, Y. Yu, and M. Mo, "A repeatable assembling and disassembling electrochemical aptamer cytosensor for ultrasensitive and highly selective detection of human liver cancer cells," Analytica Chimica Acta, vol. 885, pp. 166-173, 2015.

[45] D. Sun, J. Lu, Y. Zhong et al., "Sensitive electrochemical aptamer cytosensor for highly specific detection of cancer cells based on the hybrid nanoelectrocatalysts and enzyme for signal amplification," Biosensors and Bioelectronics, vol. 75, pp. 301307,2015

[46] F. V. Chisari, P. Filippi, J. Buras et al., "Structural and pathological effects of synthesis of hepatitis B virus large envelope polypeptide in transgenic mice," Proceedings of the National Academy of Sciences of the United States of America, vol. 84, no. 19, pp. 6909-6913, 1987.

[47] M. Fang, C.-W. Peng, S.-P. Liu, J.-P. Yuan, and Y. Li, "In vitro invasive pattern of hepatocellular carcinoma cell line HCCLM9 based on three-dimensional cell culture and quantum dots molecular imaging," Journal of Huazhong University of Science and Technology [Medical Sciences], vol. 33, no. 4, pp. 520-524, 2013.

[48] X. Fang and W. Tan, "Aptamers generated from cell-SELEX for molecular medicine: a chemical biology approach," Accounts of Chemical Research, vol. 43, no. 1, pp. 48-57, 2010.

[49] X. L. Tang, Y. Hua, Q. Guan, and C. H. Yuan, "Improved detection of deeply invasive candidiasis with DNA aptamers specific binding to $(1 \rightarrow 3)-\beta$-D-glucans from Candida albicans," European Journal of Clinical Microbiology \& Infectious Diseases, vol. 35, no. 4, pp. 587-595, 2016. 

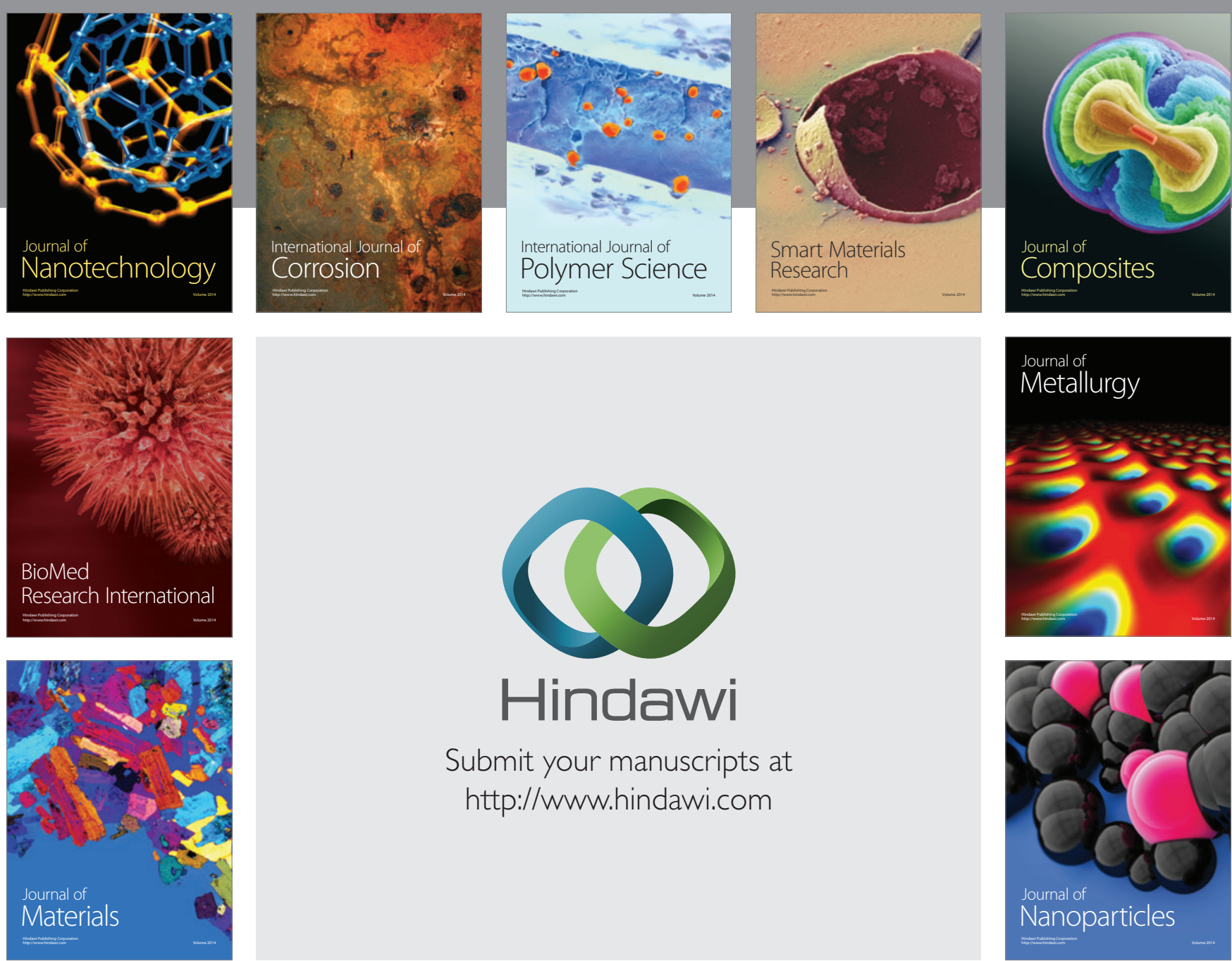

\section{Hindawi}

Submit your manuscripts at

http://www.hindawi.com

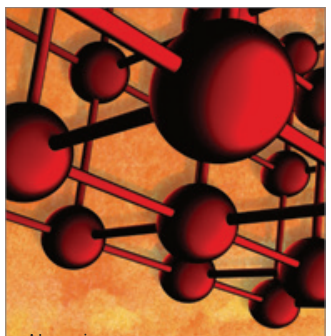

Materials Science and Engineering
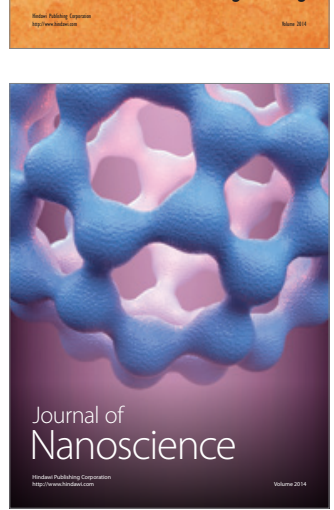
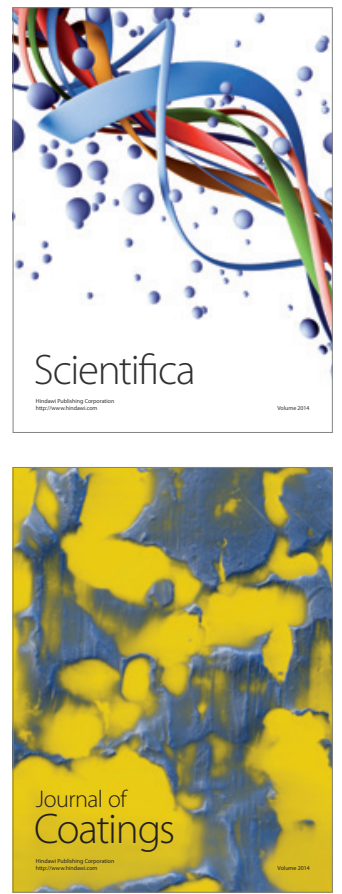
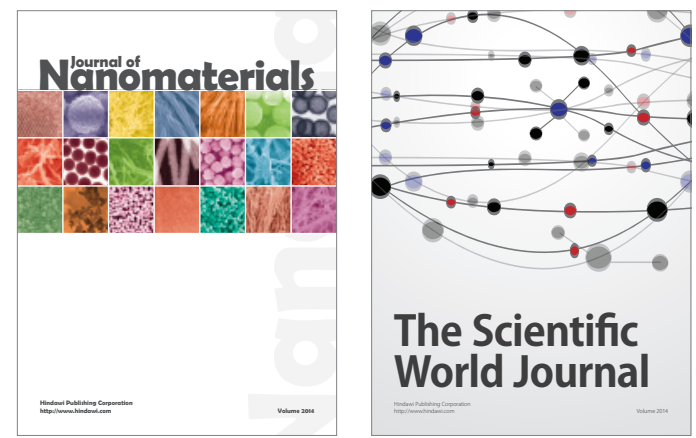

The Scientific World Journal
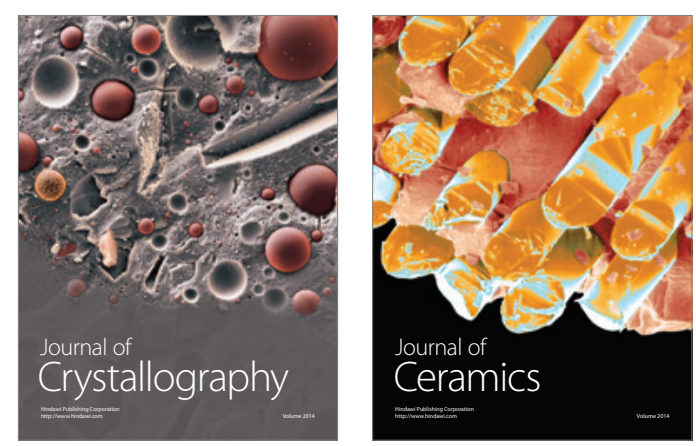
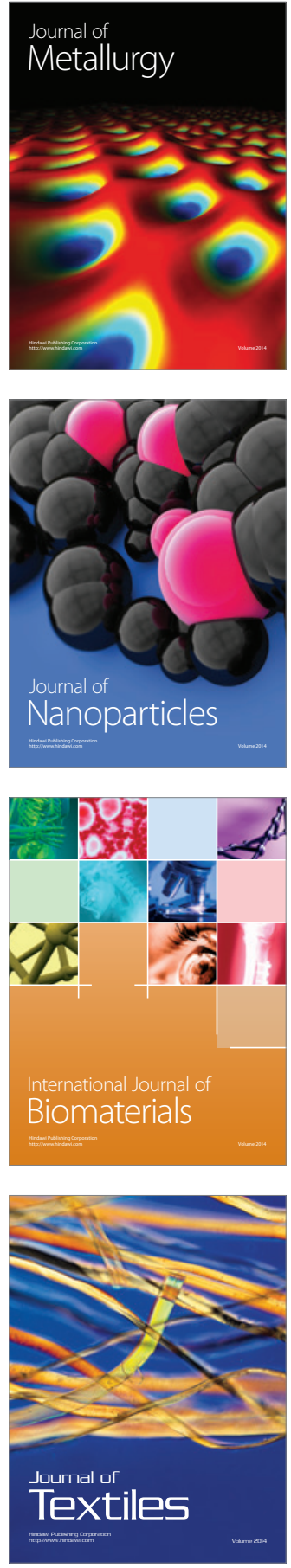